\title{
A Study on Loyalty in Science and Technology Translation
}

\author{
Xia Liu \\ Zhengzhou University of Industrial Technology, Zhengzhou 451150, China
}

Keywords: loyalty, science and technology translation, absolute loyalty.

\begin{abstract}
This paper focuses on importance of the loyalty, types of the loyalty, strategies of faithful translation and interferences of absolute loyalty in science and technology translation. In terms of importance of the loyalty in translation, this paper probes into the roles and the embodiment of loyalty in practice; Then centering on types of the loyalty, this paper analyses the translator's pursuit of perfect translation; Next, in light of strategies of faithful translation and with a large amount of supporting examples, it points out that science and technology translation activities are carried out with specific aims. Meanwhile the principles of faithfulness are feasible but have limitations. In the end, an analysis is given about interferences of absolute loyalty in English and Chinese translation to expound that pursuit of loyalty is faced with the obstacles. At last this paper reveals that there is no absolute loyalty in translation. This paper is hoped for helping people understand and master the skills of translation and to enrich the database of science and technology translation.
\end{abstract}

\section{Introduction}

Along with the swiftly current development of the science and technology, people's living standards have been amazingly improved. Thus, the products in our daily lives are required to step onto a whole new level, including electrical apparatus, professional machines and tools as well as transportation means, which manifests one of the greatest potential driving force of human kindscience and technology. Therefore, it emphasizes the importance of understanding those new and imported technologies and techniques. And how to ensure the accurate translation of these scientific and technological texts directly relates to seeable economic benefits. Then it is quite significant to grasp the language specialties of scientific and technological texts as well as the skills for translation.

\section{Importance of Loyalty in Science and Technology Translation}

Science and technology translation has brought great significance to people's daily life, academic scholars of both home and abroad are also raising their attention on this kind of translation. In the English-Chinese translation practice, there is a guiding principle for the translation process and a standard for determining the quality of the translated works, which is loyalty. Its goal is not to prove faithful and natural requirements for the translation. So we must be faithful to the form and content of the original in the process of translation. It means that the translation must convey thoughts of the original fully and the style of writing in the translation must be the same as the original text. But there exist lots of translators with different backgrounds, which make the translation more complicated. Therefore, after understanding and mastering the factors and skills of translation, we will do much better. In a word, to be a qualified translator, we must be faithful to the original. So loyalty plays an important role in English-Chinese translation.

\section{Types of the Loyalty in Science and Technology Translation}

The success and failure of the translation not only depends on its elegant language, but also relates to whether it is faithful to the original. The paper with different subjects demands the different types of the loyalty. It is roughly divided into three aspects: (1) Loyalty of style (2) Loyalty of meaning (3) Loyalty of function. Then, by analyzing the types of the loyalty in science and technology translation, it will be helpful to understand that loyalty is feasible but it has limitations in a sense. 


\subsection{Loyalty of Style}

Compared with other types of works such as news, novel, prose, practical writing, argumentation, etc., the translation of science and technology has its unique features, namely stressing on accuracy, nature and plainness, because it is an objective statement of truth. Technology text requires that the language be concise, nature and plain and it be consistent to the original style. In the meantime, it reflects the character of the original.

Science and technology articles are full of technical terms, laying stress on facts, explaining profound theories in simple language on the basis of keeping the original style, etc. Considering these high requirements of scientific writings, translators must pay attention to choose accurate and precise words rather than take too many rhetorical and modified expressions.

\subsection{Loyalty of Meaning}

Translation should express the meaning of the original. Moreover, it is based on the specific situation of the original. The following factors should be taken into consideration: people, language, circumstances, purpose. Only in this way can we reach the loyalty of meaning in the original. Loyalty of meaning is different from the loyalty of style. It further exposes the meaning that the original author wants to convey. As for science and technology translation, it first stressed on equivalence in meaning. In science and technology writings there are many technical terminologies. Each scientific and technological term could possess plural version of Chinese meanings. Each mistake in choosing the inappropriate translation word of the term can cause a huge confusion between the receivers of the target text. For instance, in the area of mechanical and electrical engineering, "bypass valves, heat exchanger, short-circuited, etc” are translated as “旁通阀、换热器、电流短路等”. There are quite a number of terms together describing a situation of how by-pass valves and heat exchanger works in this subject. In science and technology translation, the loyalty of meaning goes first.

Because the translation is a very complicated activity, the "equivalence" is only a kind of the ideal state. Frankly speaking, it isn't easy to make the translation be absolute loyal to the original.

\subsection{Loyalty of Function}

Functional loyalty highlights the function of language as a communicative tool. It is inseparable with faithful of meaning. Function is the embodiment of transcript effect, which realizes by expression of the meaning of the translation. Functional loyalty implies that the translation on the content, form, style and thoughts should be consistent with the original as far as possible. Moreover, the features of the original translation should be the same as the target language. The British Newmark finds that language has six functions: (1) Informative function, which gives information about facts; (2) Interpersonal function, which establishes and maintains social status in a society Such as age, sex, language, background, status, accent; (3) Performative function is that language is used to do things and to perform certain actions, such as promise, apologize, sorry etc; (4) Emotive function, which expresses strong feelings and profound thoughts of the speaker and author to stir the readers emotion; (5) Phatic communion, which reflects the relationship of the communicator by using some small and meaningless expression to establish a comfortable relationship or maintain social contact between people without any factual content, just like weather and health; (6) Metalingual function, which talks about language itself. In a text or a paragraph, it often can be run through a variety of functions of language, but in different types of the text, some language functions play a leading role, while some language functions play a supplementary role. Therefore, the translator should excavate the original and analyze its function when he or she does some translation. Only in this way can translators better express the original meaning and be loyal to the original, which makes the readers of the target text have the same feelings and attitudes just as the original readers.

\section{Strategies of the Loyalty in Science and Technology Translation}

When it comes to the translation strategies, we know much about them. Translation theorists come up with kinds of strategies. To sum up, there are two types of translation: (1) literal translation and 
free translation (2) foreignizing translation and domesticating translation. In this chapter, there is a detailed analysis on the combination of these translation methods with the translation cases.

\subsection{Literal Translation and Free Translation}

Both literal translation and free translation are the basic ways of the English-Chinese translation. Whether literal translation or free translation, they should meet the criteria of "faithfulness and smoothness". The purpose of literal translation and free translation is to express the original by the faithful ways. To put the loyalty of the original content in the first place, the faithful to the original form in the second place and the smooth translation form in the third are called literal translation. In contrast, to put the original content in the first place and the smooth translation form in the second place but not to adhere to the form of original are called free translation. Literal translation is in common with free translation when the translation form and the original form are consistent. In science and technology translation, the equivalence in meaning and content is of great importance. It stresses on accuracy - the correct translation of each technological terms and the correct translation of its logic connections.

For example:

The pedal pod should contact the crank arm only at its mounting face, because this is the load-bearing connection. If the pedal pod contacts the crank arm anywhere else, the pedal pod may fracture when the pedal is tightened.

踏板感应片只能与曲柄臂的安装面接触, 因为这是一个承重连接。若踏板感应片与曲柄 臂在安装面以外的地方接触, 踏板收紧时, 可能会压断踏板感应片。

The example mentioned above comes from a product manual about the vector. The target language correctly conveyed the original meaning to the consumers with the method of literal translation and such translation realizes the aim of communication adhere to the principle of loyalty.

Meanwhile, as a qualified translator, it is necessary to avoid word-for-word translation because it is the word as a unit to make every word of the original works equivalent to the transcript. On the whole, the form is very loyal and the content is quite smooth. Actually, there is as different as chalk and cheese in the meaning and style, and many readers feel unintelligible or even don't know what to say. In order to keep the translation faithful to the original, translators should also value the processing of meaning and syntactic, logic relationship of the context, the word order of the long sentence and adjustment of the sentence in the length, cultural background, etc. In addition, literal translation and free translation can be used simultaneously in the process of translation to create the language of the loyal translation.

All in all, whether literal translation or free translation, translators act according to circumstances and stick to the principle of the loyalty.

\subsection{Domesticating Translation and Foreignizing Translation}

Historically, foreignizing translation and domesticating translation can be considered as an extension of the concept of literal translation and free translation. What literal translation and free translation focus on is how to deal with the form and meaning in language level, but foreignization and domestication break through the language factors and make the horizons transfer to the factors, such as language, culture and aesthetics.

The so-called domesticating translation refers to the translation methods with the destination of the source culture. It is a little similar to the traditional principles of translation "smooth translation". Domesticating translation, essentially, makes the translation contextual, which attaches importance to the response of the target language readers.

In spite of its realistic style of science and technology texts, translators cannot neglect its aesthetic functions. For the sake of that these factors do make influence on receivers of target text to percept the aesthetic, linguistic style, the translator's personality, state of mind and educational level etc. of source text.

For example:

There are several difficulties with the by-pass valves used in the past. With some types of valves, when the valve closes and the by-pass flow stops, the element that senses the oil temperature and 
causes the valve actuation is either taken out of the flow path or overly exposed to the flow path. In the former case, the valve actuator loses its ability to sense accurately the oil temperature and open and close when desired. In the latter case, the valve actuator is exposed to the oil at the extreme ends of the oil operating temperature range, and this can permanently damage some types of actuators.

过去使用的旁通阀时常出现数个困难。对于某些类型的阀门, 当其处于关闭状态时, 旁 通流动停止, 感应油温引起气门驱动的零件, 不是偏离流体通道就是过于受到流动路径的损 害影响。对于这两种情况, 前者, 阀门执行器失去了准确检测油温和控制阀门所需的开关状 态的功能。后者, 阀门执行器容易受到极端的工作油温的影响, 便对对某些类型的传动装置 造成永久性损害。

With the effort of achieving the beauty of scientific and technological texts, the translator tries to make the linguistic style into more formal and academic. For "With some types of valves, when the valve closes and..." the translator handled the expression with more written and explanatory style. Therefore, “is off” is translated into “处于关闭状态时”。“In the former case" and “in the latter case” can be translated into “在前一种情况下” and “在后一种情况下”. Yet the translator chooses to simplify them into “对于这两种情况、前者、后者”。

In a word, the translators from various schools hold to the principle of loyalty. Loyalty is diversified multidimensional and dynamic characteristics. Translators adopt the strategy of foreignizing translation or domesticating translation or both based on the faithfulness of loyalty. Domesticating translation can make the translation smooth and easy to be understood, which increase the readability of the translation. Foreignizing translation can widen the English-Chinese in common, eliminate the gap between Chinese and English in some specific expression and help to use for reference between Chinese and English. Furthermore, it can enrich the language of the importer the translation and make the translation readers understand the culture of the source language.

\section{Conclusion}

For science and technology translation, the scope of knowledge to understand the technical expertise is the most common barrier for translators. This not only acquires us to lay a firm foundation of translation theories, bus also the preparation works to learn and understand the whole set of know-how concerning the topics of the target text. Otherwise, even if one knows the meaning of all the words in a scientific and technological text, he or she still cannot grasp the main content or the logic mainstream of the project. For every translator, he or she goes to great lengths to make his translation faithful to the source text. In fact, loyalty in translation is the basis of creative translation. What's more, loyalty is an attitude of the translator, or even a kind of desire. Sometimes, we can find that the translation seems not to be faithful to the original when we change the form of the translation, but it looks much more faithful from the whole translation.

As a translator, he or she should learn to be flexible because there exists different cultures, different states of mind, different personalities etc. However, translators still keep the translation loyalty to the original as far as possible. It is inadvisable that the translation script is partly faithful to the original, but the translators have no choice but to do it in a sense. If the translation from the whole is faithful, a small part of the problem in the translation can be neglected. All in all, the loyalty is feasible but it has limitations in the practical translation.

\section{References}

[1]. Noida, Eugene A. Toward A Science of Translating: With Special Reference to Principles and Procedures Involved in Bible Translating [M]. Leiden: E. J. Brill, 1964.p.74.

[2]. Cat ford, J.C a Linguistic Theory of Translation [M]. Oxford: Oxford University Press, 1965.p.122-123. 
[3]. Huxley, T. H. Evolution and Ethics and Other Essays [M]. New Jersey: Princeton University Press, 1989. P.14-15.

[4]. Liu, Hanged. Ten Lectuers on Literary Transaltion [M]. Beijing: ChinaTranslation \& Publishing Corporation, 1991. P.77-78.

[5]. Nord, Christiane. Translating as a Purposeful Activity: Functionalist Approaches Explained [M]. Shanghai: Shanghai Foreign Language Education Press, 1997/2001. P.145-1146. 